\title{
ENFERMAGEM EM NEFROLOGIA E SINDROME DE BURNOUT
}

\author{
Taciana Caldas Ferreira', Sânya Pedroso de Oliveira', Regina Consolação dos Santos², Cecília Godoi Campos³, \\ Nadja Cristiane Lappann Botti ${ }^{4}$, Richardson Miranda Machado ${ }^{5}$
}

RESUMO: Estudo prospectivo e exploratório foi realizado no setor de nefrologia de hospital de grande porte de Minas Gerais no período de agosto a dezembro de 2010 com os objetivos de investigar as dimensões da Síndrome de Burnout, identificar e avaliar os fatores de risco relacionados à sua ocorrência. A amostra foi constituída de 60 profissionais de enfermagem; os dados foram coletados por meio de instrumento semiestruturado e do inventário de Maslach e Jackson, e analisados a partir de suas dimensões. Dois profissionais (2,3\%) apresentaram diagnóstico de Síndrome de Burnout, 13 (22\%) apresentaram uma dimensão alterada e 43 (71,6\%) não apresentaram dimensão alterada. Gênero, escolaridade, carga horária e tempo de trabalho na instituição e ausência de outro vínculo empregatício foram considerados possíveis fatores de risco para a síndrome. A detecção precoce contribui para limitar danos decorrentes da síndrome e para a qualidade de vida do trabalhador. PALAVRAS-CHAVE: Esgotamento profissional; Enfermagem; Nefrologia; Epidemiologia.

\section{NEPHROLOGY NURSING AND BURNOUT SYNDROME}

\begin{abstract}
This prospective and exploratory study was carried out in the nephrology department of a high-capacity hospital in Minas Gerais between August and December 2010, with the objectives of investigating the scale of burnout syndrome and identifying and evaluating the risk factors related to its occurrence. The sample was made up of sixty nursing professionals and the data was collected via a semi-structured instrument and the Maslach Burnout Inventory, and analyzed from its dimensions. Two healthcare professionals (2.3\% of the sample) had a diagnosis of Burnout Syndrome. Thirteen ( $22 \%)$ had one altered dimension, and 43 (71.6\%) presented no altered dimensions of burnout. Sex, schooling, work hours, length of time at the institution and lack of another employment link were considered possible risk factors for the syndrome. Early detection contributes to limiting the harm which results from the syndrome, and contributes to the worker's quality of life.

KEYWORDS: Professional exhaustion; Nursing; Nephrology; Epidemiology.
\end{abstract}

\section{ENFERMERÍA EN NEFROLOGÍA Y SÍNDROME DE BURNOUT}

RESUMEN: Estudio prospectivo y exploratorio realizado en el sector de nefrología de un gran hospital de Minas Gerais en el periodo de agosto a diciembre de 2010 con los objetivos de investigar las dimensiones de la Síndrome de Burnout, identificar y evaluar los factores de riesgo relacionados a su ocurrencia. La muestra fue compuesta de 60 profesionales de enfermería; los datos fueron recogidos por medio de instrumento semiestructurado y del inventario de Maslach y Jackson, y analizados a partir de sus dimensiones. Dos profesionales (2,3\%) presentaron diagnóstico de Síndrome de Burnout, 13 (22\%) presentaron una dimensión alterada y 43 (71,6\%) no presentaron dimensión alterada. Género, escolaridad, carga horaria y tiempo de trabajo en la institución y ausencia de otro vínculo de trabajo fueron considerados posibles factores de riesgo para la síndrome. La detección precoz contribuye para limitar daños causados por la síndrome y ayuda en la cualidade de vida del trabajador. PALABRAS-CLAVE: Agotamiento profesional; Enfermería; Nefrología; Epidemiología.

\footnotetext{
*Artigo extraído do projeto "De bem com a Enfermagem" - Saúde Mental e Ocupacional do Profissional de Enfermagem, do Curso de Enfermagem da Universidade Federal de São João Del Rei - UFSJ.

${ }^{1}$ Acadêmica de Enfermagem da UFSJ.

${ }^{2}$ Enfermeira Executiva do Hospital São João de Deus.

${ }^{3}$ Enfermeira do Centro Mineiro de Toxicomania/ Fundação Hospitalar do Estado de Minas Gerais. Mestre em Saúde Mental.

${ }^{4}$ Enfermeira. Psicóloga. Doutora em Saúde Mental. Professora do Departamento de Enfermagem da UFSJ.

${ }^{5}$ Enfermeiro. Doutor em Saúde Mental. Professor da UFSJ. Orientador.
}

Autor correspondente:

Recebido: $14 / 10 / 2011$

Taciana Caldas Ferreira

Aprovado: 14/02/2012

Universidade Federal de São João Del Rei

Rua Canápolis, 690 - 35501-229 - Divinópolis-MG-Brasil

E-mail: tacicaldas@yahoo.com.br

Cogitare Enferm. 2012 Jan/Mar; 17(1):44-9 


\section{INTRODUÇÃO}

A Síndrome de Burnout é definida como um transtorno psíquico decorrente da forte tensão emocional crônica associada ao estresse ocupacional severo. Verificase uma alta incidência entre profissionais responsáveis pela assistência às pessoas que necessitam de algum tipo de cuidado e/ou ajuda, com função assistencial caracterizada pelo relacionamento direto e frequente ${ }^{(1)}$.

As manifestações dessa Síndrome incluem a perda progressiva do idealismo, das expectativas, da energia, da satisfação e do comprometimento com a função de cuidado. Esses sintomas indicam a presença de um autoconceito negativo e resultam na ocorrência de prejuízo ao trabalho e na presença de atritos com as pessoas que se encontram no ambiente laboral ${ }^{(1)}$.

Neste sentido, a Enfermagem, cuja essência como profissão é o cuidar, configura-se como uma das profissões mais acometidas pela Síndrome de Burnout, devido à frequente sobrecarga de trabalho, muitas vezes justificada pela falta de pessoal e/ou estimulada pelo pagamento de horas-extras e a falta de autonomia e autoridade na tomada de decisões, entre outros motivos, o que gera um estado de estresse crônico. Deste modo, a deterioração da qualidade da assistência e os altos índices de absenteísmo dos profissionais dessa área são algumas das consequências deste quadro ${ }^{(2-3)}$.

O estresse relacionado ao trabalho reduz a produtividade, a qualidade e a eficiência da assistência promovida pelo profissional de enfermagem, prejudicando significativamente o processo de recuperação e cura dos pacientes. Isso se torna ainda mais grave ao considerarmos os setores fechados dos hospitais, onde se encontram pacientes com problemas de saúde mais graves, debilitados, necessitando da assistência de enfermagem contínua e intensiva.

Os setores hospitalares como os da nefrologia, são considerados como setores fechados, pois a circulação de profissionais, familiares e visitantes é limitada, uma vez que se encontram pacientes em estado grave de saúde e/ou sendo submetidos a procedimentos delicados. O cuidado de enfermagem prestado nestas unidades é considerado exaustivo e tenso, transformando-se em desgaste físico e mental. Assim, pode-se repercutir em acometimento pela Síndrome de Burnout e, consequentemente, em absenteísmo laboral, licenças médicas e risco de acidentes de trabalho(4).

Partindo destes pressupostos teóricos, este estudo teve como objetivo investigar as dimensões da Síndrome de Burnout nos profissionais de enfermagem do setor de Nefrologia de um hospital de grande porte da região centro-oeste de Minas Gerais. Assim, reconhecendo-se a importância da qualidade de vida no trabalho, para que os profissionais de enfermagem exerçam o cuidado humanizado e consigam atuar com qualidade nos serviços de saúde, sem prejuízo para a sua qualidade de vida.

\section{MÉTODO}

Trata-se de um estudo prospectivo e exploratório realizado no setor de Nefrologia de um hospital de grande porte localizado na cidade de Divinópolis-MG, município considerado referência pelo SUS para a região Centro-Oeste de Minas Gerais. O hospital é classificado como de cuidado terciário e filantrópico, com capacidade total instalada de 300 leitos, sendo que $75 \%$ dos seus pacientes são atendidos pelo SUS e, em sua grande maioria, provenientes dos 54 municípios da região Centro-Oeste Mineira. O setor de Nefrologia possui 40 máquinas de hemodiálise, 29 leitos móveis e realiza cerca de 2400 procedimentos dialíticos ao mês ${ }^{(4)}$.

Para participarem da pesquisa, os funcionários foram selecionados a partir do critério de inclusão - ser funcionário da Nefrologia há, no mínimo, três meses; foram considerados critérios de exclusão: preenchimento incompleto dos questionários de avaliação, estar de férias, afastamento ou de licença médica do trabalho.

Para o levantamento dos dados, foram utilizados dois instrumentos: o primeiro foi elaborado pelos autores da pesquisa contendo três partes: I) dados demográficos (sexo, idade, estado civil e número de filhos); II) características profissionais (titulação, turno de trabalho, tempo de experiência profissional e na instituição hospitalar, carga horária semanal no trabalho e vínculo empregatício); III) características de lazer dos profissionais (preenchimento das horas vagas e exercício de alguma atividade física).

O segundo instrumento foi o inventário de Maslach e Jackson (Maslach Burnout Inventory - MBI - versão HSS - Human Services Survey), desenvolvido para avaliar a Síndrome de Burnout em suas três dimensões e validado no Brasil $^{(5)}$.

No Brasil, o inventário MBI-HSS foi traduzido e validado a partir da verificação dos escores de Burnout em relação aos sentimentos pessoais, atitudes do profissional no trabalho, frente aos pacientes e demais profissionais da equipe de saúde. Esse inventário contém 22 questões, sendo nove ligadas à exaustão emocional, cinco à despersonalização e oito à baixa realização pessoal no 
trabalho. Cada item indica a frequência das respostas em uma escala de pontuação que vai de zero a quatro. Sendo 0 para "nunca", 1 para "algumas vezes ao ano", 2 para "algumas vezes ao mês", 3 para indicar "algumas vezes na semana" e 4 para indicar "diariamente"(5).

O Burnout é caracterizado por três dimensões, que são avaliadas em índices altos e baixos. A primeira dimensão é a exaustão emocional, na qual o contato frequente e intenso com pessoas que vivem em situações de sofrimento gera uma enorme carga emocional. O profissional sente-se esgotado, sem energia, pouco tolerante, facilmente irritável e nervoso no ambiente de trabalho. Considerou-se nível alto para exaustão emocional quando a soma das pontuações citadas acima foi maior ou igual a 27, nível moderado quando a soma ficou entre 19 e 26 e nível baixo quando foi menor que $19^{(5)}$.

A segunda é a despersonalização, na qual o profissional assume uma atitude desumana. Progressivamente, ocorre um distanciamento emocional, que traz frieza e indiferença diante das necessidades dos outros. Ele perde a capacidade de identificação e empatia com as pessoas que precisam de ajuda e não as trata como seres humanos e, sim, como objetos. Para despersonalização, a soma maior ou igual a 10 significou um nível alto, o nível foi considerado moderado quando a soma ficou entre $06 \mathrm{e}$ 09 e nível baixo para a soma menor que $06^{(5)}$.

A terceira é o minoramento da realização pessoal e profissional. No decorrer do tempo, desenvolve-se um sentimento de decepção e frustração por não estar desenvolvendo o que tinha planejado para a sua vida, sonhos, ambições $^{(5)}$. Para a realização profissional, o nível alto foi considerado quando a soma foi menor ou igual a 33 , se o somatório ficou entre 34 e 39 considerou-se nível moderado e nível baixo quando a somatória for maior ou igual a 40.

O manual do MBI-HSS traz como princípio para o diagnóstico a obtenção de nível alto para exaustão emocional e despersonalização e nível baixo para realização profissional. Portanto, o enquadramento do profissional nesses três critérios dimensionais indica a manifestação da síndrome ${ }^{(5)}$. Para a interpretação da pontuação do inventário MBI-HSS foram utilizados tanto os segmentos teóricos que definem a Síndrome pela presença das três dimensões em níveis altos, quanto os que aceitam apenas uma, independente de qual seja, para fazero diagnóstico ${ }^{(5-7)}$.

Os dados foram coletados no segundo semestre do ano de 2010, por um único pesquisador, em sala reservada e anexa ao setor, após o projeto ser aprovado pelo Comitê de Ética em Pesquisa do HSJD (protocolo n. 34/2010).

Após o término da aplicação dos questionários, as informações foram extraídas desses e inseridas nos softwares Microsoft Excel $^{\mathbb{}}$ - versão 2003 e Statistical Package for the Social Sciences (SPSS) - versão 13.0, os quais permitiram o tratamento das variáveis coletadas e a apresentação dos resultados por meio de estatística descritiva.

\section{RESULTADOS}

Participaram do estudo 60 profissionais, entre enfermeiros, técnicos e auxiliares de enfermagem, caracterizados na tabela 1 de acordo com as dimensões da Síndrome de Burnout e a variável sexo.

Pode-se observar na tabela 1 que houve a predominância do sexo feminino no setor, com 52 profissionais (87\%); quatro possuíam índice alto e 48 baixo para exaustão emocional. Apresenta-se, também, a ocorrência das dimensões da Síndrome, de acordo com a

Tabela 1 - Caracterização dos profissionais de enfermagem nas dimensões da Síndrome de Burnout segundo o gênero, idade e estado civil. Divinópolis, 2010

\begin{tabular}{lcccccc}
\hline \multicolumn{1}{c}{ Sexo } & \multicolumn{2}{c}{ Exaustão Emocional } & \multicolumn{2}{c}{ Despersonalização } & \multicolumn{2}{c}{ Realização Profissional } \\
& Alto & Baixo & Alto & Baixo & Alto & Baixo \\
\hline Feminino & $4(6,7 \%)$ & $48(80 \%)$ & $9(15 \%)$ & $43(71,7 \%)$ & $8(13,3 \%)$ & $44(73,3 \%)$ \\
Masculino & $1(1,7 \%)$ & $7(11,7 \%)$ & $1(1,7 \%)$ & $7(11,7 \%)$ & $1(1,7 \%)$ & $7(11,7 \%)$ \\
$\quad$ Idade & & & & & & \\
20 a 30 & $1(1,6 \%)$ & $19(31,7 \%)$ & $3(5 \%)$ & $17(28,3 \%)$ & $4(6,7 \%)$ & $16(26,7 \%)$ \\
31 a 40 & $1(1,6 \%)$ & $17(28,3 \%)$ & $2(3,3 \%)$ & $16(26,7 \%)$ & $3(5 \%)$ & $15(25 \%)$ \\
41 a 50 & $3(5 \%)$ & $16(26,7 \%)$ & $5(8,3 \%)$ & $14(23,3 \%)$ & $3(5 \%)$ & $16(26,7 \%)$ \\
> 50 anos & $0(0 \%)$ & $3(5 \%)$ & $1(1,7 \%)$ & $2(3,3 \%)$ & $1(1,7 \%)$ & $2(3,3 \%)$ \\
Estado Civil & & & & & & \\
Solteiro & $1(1,7 \%)$ & $25(41,7 \%)$ & $4(6,7 \%)$ & $22(36,7 \%)$ & $4(6,7 \%)$ & $22(36,7 \%)$ \\
Casado & $4(6,7 \%)$ & $26(43,3 \%)$ & $5(8,3 \%)$ & $25(41,7 \%)$ & $4(6,7 \%)$ & $26(43,3 \%)$ \\
Divorciado & $0(0 \%)$ & $4(6,7 \%)$ & $1(1,7 \%)$ & $3(5 \%)$ & $1(1,7 \%)$ & $3(5 \%)$ \\
\hline
\end{tabular}

Cogitare Enferm. 2012 Jan/Mar; 17(1):44-9 
faixa etária dos profissionais. E, ainda, verificou-se que $50 \%$ dos funcionários eram casados (30), variando as dimensões da Síndrome de acordo com o estado civil dos participantes.

Entre os que não possuíam filhos, ao analisar a exaustão emocional, quatro obtiveram índice alto e 24 obtiveram um índice baixo; na despersonalização o mesmo valor, quatro e 24 para índice alto e baixo, respectivamente. Ao verificar a realização profissional nota-se que cinco funcionários obtiveram índice alto e 23 índice baixo.

Ao associar as dimensões de Burnout com o turno de trabalho, percebeu-se que 16 funcionários (27\%) trabalhavam durante o turno matutino, onde 3 alcançaram índice alto e 13 índice baixo para exaustão emocional e despersonalização; já na realização profissional, dois funcionários obtiveram índice alto e 14 índice baixo.No turno diurno, trabalhavam 9 funcionários (15\%). Ao analisar a exaustão emocional e a realização profissional, os 9 funcionários obtiveram índice baixo, enquanto que na despersonalização somente um obteve índice alto e 8 obtiveram índice baixo. Entre 12 funcionários $(20 \%)$ que trabalhavam durante o turno vespertino, a exaustão emocional de todos teve índice baixo; na despersonalização, um funcionário teve índice alto e 11, índice baixo; e na realização profissional, um possuiu índice alto e 11 índice baixo.

Registraram-se no período noturno 23(38\%) funcionários, sendo que, na exaustão emocional 21 tiveram índice baixo e dois alto; 18 tiveram índice baixo e cinco índice alto para despersonalização; já na realização profissional, 17 obtiveram índice baixo e seis índice alto.

Podemos observar, analisando os dados do setor, que a maioria foi constituída de profissionais que possuíam o ensino médio 49(81,7\%), seguido da graduação 6(10\%) e $5(8,3 \%)$ com a titulação de pós-graduação.

Nota-se ao avaliar os profissionais com a titulação de ensino médio que cinco tiveram índice alto e 44 índice baixo para exaustão emocional, na despersonalização, dez obtiveram um índice alto e 39 um índice baixo, já na realização profissional, nove tiveram índice alto e 40 índice baixo. Todos os funcionários graduados obtiveram o mesmo nível baixo, seis. O mesmo aconteceu com os funcionários com pós-graduação.

Na tabela 2 avalia-se o tempo de trabalho na instituição, sendo que a maioria dos profissionais trabalhavam na instituição há menos de cinco anos (40\%). Esses possuíam um nível baixo para exaustão emocional, para a despersonalização há quatro com índice alto e 20 com índice baixo, o mesmo valor foi encontrado na realização profissional.

Já para os profissionais que trabalhavam de seis a dez anos na instituição, percebe-se que 3 possuíam índice alto e 8 com índice baixo para a exaustão emocional, na despersonalização 2 profissionais possuíam índice alto e 9 com índice baixo. Notamos na realização profissional os mesmos valores que os obtidos na despersonalização. Para os funcionários que trabalhavam de 11 a 15 anos na instituição, os valores são os mesmos para a exaustão emocional, despersonalização e realização profissional, sendo 9 com índice baixo e nenhum com índice alto. Entre os profissionais que trabalhavam na instituição de 16 a 20 anos, 2 possuíam índice alto para exaustão emocional e 9 nível baixo para o mesmo. Na despersonalização, 4 apresentaram índice alto e 7 índice baixo, já na realização profissional, 3 índice alto e 8, índice baixo.

Para os profissionais que trabalhavam há mais de 20 anos, 1 obteve índice alto para exaustão emocional e 4 obtiveram índice alto para essa dimensão. Para despersonalização e realização profissional os índices foram os mesmos, nenhum alto e 5 baixos.

A maioria dos funcionários não possuía outro emprego (82\%), variando a ocorrência das dimensões da Síndrome, conforme a tabela 2.

Tabela 2 - Caracterização dos profissionais de enfermagem nas dimensões da Síndrome de Burnout segundo o tempo de trabalho e vínculo empregatício. Divinópolis, 2010

\begin{tabular}{lcccccc}
\hline \multirow{2}{*}{ Tempo de trabalho } & \multicolumn{2}{c}{ Exaustão Emocional } & \multicolumn{2}{c}{ Despersonalização } & \multicolumn{2}{c}{ Realização Profissional } \\
& Alto & Baixo & Alto & Baixo & Alto & Baixo \\
\hline 0 a 5 anos & $0(0 \%)$ & $24(40 \%)$ & $4(6,7 \%)$ & $20(33,3 \%)$ & $4(6,7 \%)$ & $20(33.3 \%)$ \\
6 a 10 anos & $3(5 \%)$ & $8(13,3 \%)$ & $2(3,3 \%)$ & $9(15 \%)$ & $2(3,3 \%)$ & $9(15 \%)$ \\
11 a 15 anos & $0(0 \%)$ & $9(15 \%)$ & $0(0 \%)$ & $9(15 \%)$ & $0(0 \%)$ & $9(15 \%)$ \\
16 a 20 anos & $2(3,3 \%)$ & $9(15 \%)$ & $4(6,7 \%)$ & $7(11,7 \%)$ & $3(5 \%)$ & $8(13,3 \%)$ \\
> 20 anos & $1(1,7 \%)$ & $4(6,7 \%)$ & $0(0 \%)$ & $5(8,3 \%)$ & $0(0 \%)$ & $5(8,3 \%)$ \\
\multicolumn{2}{r|}{ Possui outro emprego } & & & & & \\
Sim & $0(0 \%)$ & $11(18,3 \%)$ & $0(0 \%)$ & $11(18,3 \%)$ & $0(0 \%)$ & $11(18,3 \%)$ \\
Não & $5(8,3 \%)$ & $44(73,3 \%)$ & $10(16,7 \%)$ & $39(65 \%)$ & $9(15 \%)$ & $40(66,7 \%)$ \\
\hline
\end{tabular}




\section{DISCUSSÃO}

A partir da amostra estudada, obteve-se 2(3,3\%) diagnósticos da Síndrome de Burnout, ambos do gênero feminino, com a mesma titulação (ensino médio), carga horária semanal, com tempo de trabalho na instituição de 06 a 10 anos e sem outro vínculo empregatício.

Ao analisarmos os dados, foram identificados $13(21,7 \%)$ profissionais que apresentaram somente uma dimensão para a síndrome e outros 3(5\%) que apresentaram duas dimensões alteradas. Esses casos, nos quais se percebeu a alteração de pelo menos uma das dimensões, merecem cuidado ocupacional e organizacional preventivo de maior qualidade, para que se possa evitar o avanço da Síndrome.

A amostra foi, em sua maioria, composta por mulheres, na faixa etária entre 20 e 30 anos de idade, casadas, com filhos e que exercem suas atividades laborais durante o turno noturno. Ainda, trabalhadoras com titulação de ensino médio, sem outro vínculo empregatício e com tempo de trabalho na instituição abaixo de cinco anos.

Estudos $^{(8-9)}$ demonstram que ser profissional mulher é fator predisponente à Síndrome de Burnout, em razão da jornada dupla de trabalho, que inclui profissão e afazeres domésticos, que na maioria das vezes as mulheres são submetidas. Profissionais casados ou que têm um companheiro fixo e os que têm filhos, possuem uma menor propensão de desenvolver Burnout ${ }^{(2,6-7)}$

Quanto à faixa etária, o presente estudo evidenciou que profissionais entre 20 e 30 anos encontravam-se com as dimensões da Síndrome de Burnout alteradas. Acredita-se que isso ocorra devido à alta expectativa frente ao trabalho e à insegurança do recém-formado frente à realidade encontrada no trabalho ${ }^{(6,8)}$.

Ao analisarmos o turno de trabalho, os profissionais do turno da noite apresentaram maior frequencia de dimensões altas para Síndrome, devido ao fato de que o sono perdido neste período, dificilmente é substituído por um de mesmo tempo e qualidade ${ }^{(2,6)}$. Vale ressaltar, que os profissionais que trabalhavam no setor estudado, mudam mensalmente o turno de trabalho, o que contribui para a prevenção da Síndrome de Burnout ${ }^{(10)}$.

Nesta pesquisa, os achados demonstram que a maioria dos profissionais não possuíam outro vínculo empregatício, o que é um fator preventivo para o desenvolvimento da Síndrome visto que o vínculo com mais de uma empresa aumenta a sobrecarga física e mental dos profissionais e, consequentemente, leva ao aparecimento dos sintomas da Síndrome. Estudos evidenciam que a carga horária semanal é uma das causas que mais evidencia o aparecimento da Síndrome nos profissionais de enfermagem, causando maior desgaste físico e emocional, visto que o tempo destinado ao lazer é inversamente proporcional ao tempo destinado ao trabalho ${ }^{(9)}$.

Os profissionais de enfermagem podem desenvolver patologias, as quais resultarão na ajuda de uma equipe de saúde e sendo que o profissional ocupa agora, a posição de paciente. A prevenção é necessária e consiste em aumentar a variedade de rotinas, reduzir e evitar o excesso de horas extras, oferecer maior e melhor suporte psicossocial aos profissionais, dando melhores condições sociais e físicas de traba1 ho ${ }^{(10)}$. A prática de atividades físicas por metade dos profissionais pode ser um fator de proteção para o desenvolvimento de Burnout.

\section{CONCLUSÃO}

A Síndrome de Burnout é uma patologia cada vez mais frequente entre os profissionais de enfermagem e requer maior divulgação como meio de prevenção de sinais e, principalmente, medidas para sua detecção precoce e prevenção. Os resultados deste estudo demonstraram que um quarto dos trabalhadores necessitavam intervenção para limitação das consequências e progressão dos sintomas.

A síndrome é um fator resultante da relação profissional - paciente e instituição, devendo ser ativamente investigada para que estratégias integradas e direcionadas para as modificações laborais sejam elaboradas, resultando em segurança e bem-estar dos trabalhadores.

\section{REFERÊNCIAS}

1. Tamayo MR, Troccoli BT. Construção e validação fatorial da escala de caracterização do burnout (ECB). Estud. psicol. 2009;14(3):213-21.

2. Moreira DS, Magnago RF, Sakae TM, Magajewski FRL. Prevalência da Síndrome de Burnout em trabalhadores de enfermagem de um hospital de grande porte da Região Sul do Brasil. Cad. saude publica. 2009;25(7):1559-68.

3. Pereira CA, Miranda LCS, Passos JP. O estresse ocupacional da equipe de enfermagem em setor fechado. R. pesq. cuid. fundam. online. 2009;1(2):196-202. 
4. Hospital São João de Deus. [Internet] Divinópolis [acesso em 29 ago 2011]. Apresentação. Disponível: http://www.hsjd.com.br/hsjd/index.asp

5. Gil-Monte PR, Carlotto MS, Câmara SG. Validação da versão brasileira do questionário para a avaliação da Síndrome de Burnout em trabalhadores. Rev. saude publica. 2010;44(1):140-7.

6. Fogaça MC, Carvalho WB, Cítero VA, NogueiraMartins LA. Fatores que tornam estressante o trabalho de médicos e enfermeiros em terapia intensiva pediátrica e neonatal: estudo de revisão bibliográfica. Rev. bras. ter. intensiva. 2008;20(3):261-6.

7. Jodas DA, Haddad MCL. Síndrome de Burnout em trabalhadores de enfermagem de um pronto socorro de hospital universitário. Acta paul. enferm. 2009;22(2):192-7.

8. Santos FE, Alves JA, Rodrigues AB. Síndrome de Burnout em enfermeiros atuantes em uma Unidade de Terapia Intensiva. Einstein. 2009;7(1 Pt 1):58-6.

9. Santos TMB, Frazão IS, Ferreira DMA. Estresse ocupacional em enfermeiros de um hospital universitário. Cogitare enferm. 2011;16(1):76-81.

10. Ruviaro MFS, Bardagi MP. Síndrome de Burnout e satisfação no trabalho em profissionais da área de enfermagem do interior do RS. Barbarói. 2010;33:194216. 